\section{Gräsertablette zeigt nachhaltige Wirksamkeit}

D ie spezifische Immuntherapie (SIT) wird von der WHO als einzige Ursachen-bekämpfende Allergiebehandlung anerkannt und zeichnet sich insbesondere durch eine langfristige Verbesserung der Lebensqualität aus. Dabei ten zur kausalen Behandlung von Heuschnupfen eine sichere und effektive Alternative zur subkutanen Immuntherapie (SCIT) dar.

Zwei Langzeitstudien zur Behandlung mit Allergie-Immuntherapie-Tabletten sollten bestätigen, dass die Beschwerden auch über die Therapiedauer hinaus gestellen Allergie-Immuntherapie-Tablet-

mindert werden. In einer Phase-III-Studie nahmen über 600 Gräserpollen-Allergiker drei Jahre lang einmal täglich entweder die Gräser-Impf-Tablette Grazax $^{\circ}$ oder ein Placebo ein [Dahl R et al. J Allergy Clin Immunol 2008; 121: 512-8]. Die Symptome wurden mittels ARTSS (,average rhinoconjunctivits total symptome score") erhoben. Die Beschwerden verbesserten sich im gesamten Behandlungszeitraum gegenüber der Vergleichsgruppe um ca. ein Drittel. Die Verträglichkeit wurde dabei als sehr gut beurteilt. Auch während der zweijährigen Nachbeobachtungsphase waren die

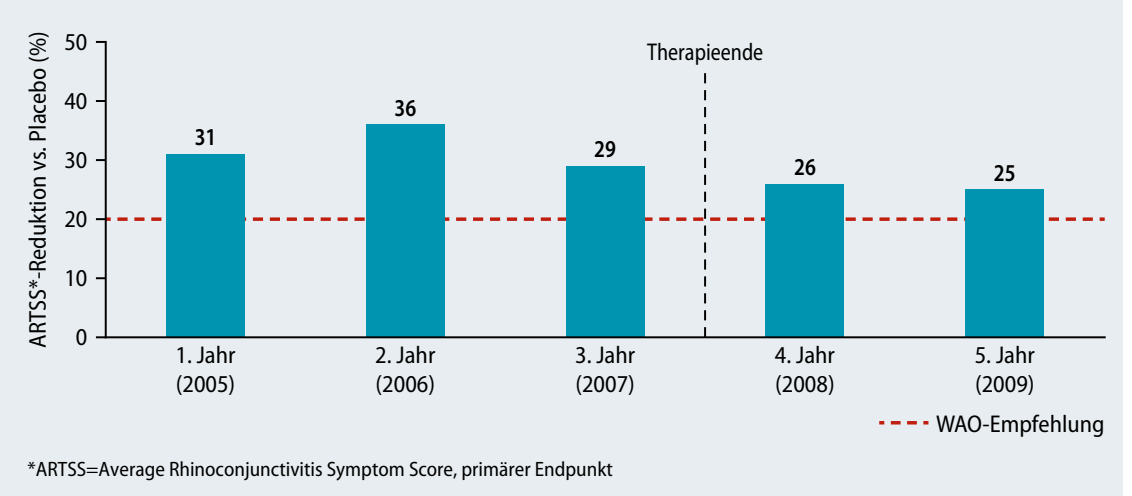

Langzeitwirksamkeit: Auch zwei Jahre nach Ende der aktiven Therapie mit Grazax ${ }^{\circledR}$ hält der Effekt noch an (nach Durham SR et al. J Allergy Clin Immunol 2012; 129: 717-25).

\section{Der präventive Marsch als Antwort auf den allergischen Marsch}

m Rahmen eines Symposiums des Unternehmens Symbiopharm, das auf dem 8. Deutschen Allergiekongress in Bochum stattgefunden hat, skizzierte Prof. Dr. Eckard Hamelmann die Vision eines „präventiven Marschs“. Analog zum „allergischen Marsch“ sollen dabei mehrere hintereinander geschaltete Maßnahmen die Entstehung einer Allergie verhindern. Bereits bei Risikokindern und noch vor dem Nachweis der ersten allergischen Sensibilisierung kann eine primäre Prävention stattfinden, z. B. durch eine unspezifische Immunmodulation über bakterielle Lysate oder über eine präventive Immuntherapie mit einem Allergencocktail. Bei Patienten, die bereits eine Atopie entwickelt
Symptome um noch ca. ein Viertel reduziert.

In einer vergleichbar großen Studie, in der die Allergentablette Oralair ${ }^{\circledast}$ prä-cosaisonal verabreicht wurde, zeigte sich eine signifikante Reduktion der Symptome nach ARTSS erst im zweiten und dritten Jahr der Therapie bei ebenfalls guter Verträglichkeit [Didier A et al. J Allergy Clin Immunol 2011; 128: 55966; Didier A et al. Oral abstract XXII World Allergy Congress, Cancún, Mexico, Dec 5, 2011]. Ein Jahr nach Behandlungsende lag der Symptomscore im Vergleich zur Placebogruppe noch um $21 \%$ niedriger. Werte für das zweite Nachbeobachtungsjahr wurden ebenfalls erhoben, aber bisher nicht veröffentlicht.

Damit ist Grazax $^{\circledast}$ derzeit die in Deutschland einzig zugelassene Allergie-Immuntherapie-Tablette, für die eine Langzeitwirksamkeit und der Disease-Modyfing-Effect bestätigt ist. Ein Vergleich der beiden Langzeitstudien zeigt zudem, dass die ganzjährige Therapie der prä-cosaisonalen Verabreichung im Hinblick auf den nachhaltigen Therapieerfolg womöglich überlegen ist [Lobermeyer K et al. Allergo J 2013; 22: 401].

Nach Informationen von ALK-Abelló

sich. Ausschlaggebend dafür ist der fehlende Kontakt des Neugeborenen zu den Bakterien des Geburtskanals.

Wie eine aktuelle Veröffentlichung zur PAPS-Studie mit 606 Säuglingen zeigte, wirken sich auch ältere Geschwister direkt auf die Diversität der Darmflora aus und schützen so vor Allergien [Pender J et al. J Allergy Clin Immunol. 2013; 132: 601-7]. Eine Besiedlung mit Clostridien zwischen der fünften und der 13. Lebenswoche wurde dagegen als ein Risikofaktor für die Entwicklung einer atopischen Dermatitis identifiziert und zeigte sich bevorzugt bei Kindern, die keine oder wenige ältere Geschwister haben und/oder per Sectio auf die Welt kamen.

Nach Informationen von Symbiopharm 